\title{
ROSAT X-ray Observations of Pair Mrk474/NGC 5682
}

\author{
Hongguang $\mathrm{Bi}$ \\ MPI für Extrater. Physik, 85748 Garching, FRG \& Beijing Obs., Beijing 100080, CHINA
}

\begin{abstract}
Like radio and optical observations of AGN/Galaxy pairs (e.g. Carilli, C \& van Gorkom, J. 1992, ApJ, 399, 373), X-ray observations of the pairs can reveal absorption at high energy band, and also, possible galactic gaseous X-ray emissions that are very useful in constructing halo models. ROSAT/PSPC X-ray spectra of 4 AGNs in the well-known pairs 3C232/NGC 3067, 3C275.1/NGC 4651, 3C309.1/NGC 5832 and Mrk474/NGC 5682 are reported here. Especially, we have detected an extragalactic HI of NGC 5862 in the Mrk474 spectrum.
\end{abstract}

Key words: AGN/Galaxy pairs, AGN X-ray spectrum, extragalactic HI

\section{Observations}

X-ray spectra of the AGNs are fitted by power law of $f(E) d E=A\left(\frac{E}{1 \mathrm{keV}}\right)^{-\Gamma} d E$ plus a neutral hydrogen absorption using $\chi^{2}$ method. The resulting column densities $N_{H I}$ are consistent with absorptions in our galaxy in all AGNs except for Mrk474, partly because the 3 3CR QSOs do not have enough counts $(\leq 300)$ for good spectral calculation. Mrk474 that has $\sim 10830$ total counts can be fitted by $N_{H I}=$ $3.61 \pm 0.23 \times 10^{20} \mathrm{~cm}^{-2}$ (comparing to the Galactic $N_{H I}=2.01 \times 10^{20} \mathrm{~cm}^{-2}$ ) and photon index $\Gamma=-2.31 \pm 0.06$. Note that the power law fitting has systematic positive residuals below $0.5 \mathrm{keV}$, another blackbody component is then added that results in even larger an $\mathrm{HI}$ of $5.5 \pm 1.0 \times 10^{20} \mathrm{~cm}^{-2}$. Therefore, an extragalactic absorption of $N_{H}=1.3 \sim 4.5 \times 10^{20} \mathrm{~cm}^{-2}$ is evidenced.

An optical image of the pair is in Fig.1a, the bar gives the angular scale of 1 arc minute (Arp, H., Baldwin, J.A. \& Wampler, E.J. 1975, ApJ, 198, L3). Fig. 1b shows a deconvolved ROSAT X-ray image. The X-ray center is coincident with M474. The nearby galaxy NGC5682 has an average $N_{H}=9.2 \times 10^{20} \mathrm{~cm}^{-2}$ within the linear diameter $13.8 \mathrm{kpc}$ (Bottinelli, L., et al. 1984, A\&AS, 56, 381). Assuming the HI radial density drops as $\sim r^{-2}$, we estimate that at the M474 impact distance $13 \mathrm{kpc}$ it is about 3 times smaller that the average : $3.1 \times 10^{20}$ $\mathrm{cm}^{-2}$, which is very close to that derived from the ROSAT X-ray spectrum.

However, the X-ray configuration of the pair shows interesting structures to be studied carefully. In Fig.1b, the emission consists of at least four component : the nucleus (M474), two sources at $\left(-48^{\prime \prime},-40^{\prime \prime}\right)$ and $\left(82^{\prime \prime},-61^{\prime \prime}\right)$ relative to M474 (the first one is identified as an optical source, marked by the arrow), and a diffuse emission at $50^{\prime \prime} \mathrm{NE}$ from M474 that may in turn be a composition of 3 or more point sources. No optical counterpart can be identified to such a diffuse feature.

Acknowledgements. I am grateful to Drs. J. Trümper and H. Zimmermann for stimulating discussions and hospitality when I visited MPE. Dr. H. Arp is the PI and Dr. J. Sulentic is another Co-I of the 4 AGN/NGC observations. I thank them for allowing me use the preliminary results here.

366

T. J.-L. Courvoisier and A. Blecha: Multi-Wavelength Continuum Emission of AGN, 366-367.

(C) 1994 IAU. Printed in the Netherlands. 


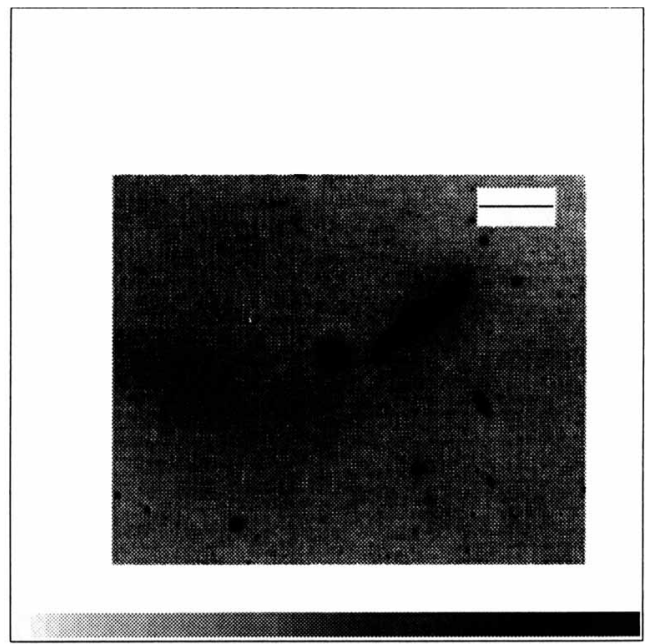

Fig_1a - Mrk474/NGC5682:optical

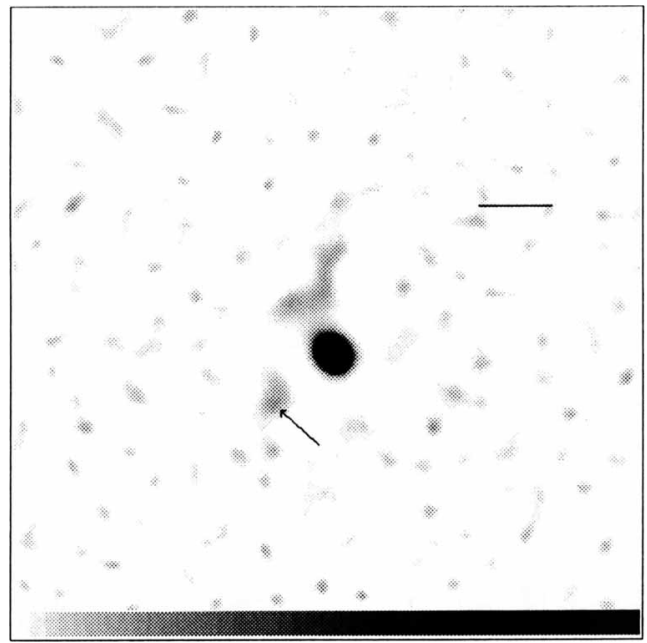

Fig_1b - Mrk474/NGC5682:deconvolution 